\title{
Evaluation of a Mathematical Model for Digital Image Enhancement
}

\author{
Hassem Geha $^{1}$, Ibrahim Nasseh ${ }^{2, *}$ and Marcel Noujeim ${ }^{3}$ \\ ${ }^{I}$ The University of Texas Health Science Center, San Antonio, United States; ${ }^{2}$ Department of Oral and Maxillofacial \\ Radiology, Lebanese University, Beirut, Lebanon; ${ }^{3}$ The University of Texas Health Science Center, San Antonio, United \\ States
}

\begin{abstract}
Objective: The purpose of this study is to compare the detected number of holes on a stepwedge on images resulting from the application of the 5th degree polynomial model compared to the images resulting from the application of linear enhancement. Material and Methods: A 10-step aluminum step wedge with holes randomly drilled on each step was exposed with three different $\mathrm{kVp}$ and five exposure times per $\mathrm{kVp}$ on a Schick $33^{\circledR}$ sensor. The images were enhanced by brightness/contrast adjustment, histogram equalization and with the 5th degree polynomial model and compared to the original non-enhanced images by six observers in two separate readings. Results: There was no significant difference between the readers and between the first and second reading. There was a significant three-factor interaction among Method, Exposure time, and $\mathrm{kVp}$ in detecting holes. The overall pattern was: "Poly" results in the highest counts, "Original" in the lowest counts, with "B/C" and "Equalized" intermediate. Conclusion: The 5th degree polynomial model showed more holes when compared to the other modalities.
\end{abstract}

Keywords: Contrast, digital imaging, histogram, image enhancement.

\section{INTRODUCTION}

Digital image technology offers a multitude of options for improving the visual quality of diagnostic images, with the most appropriate enhancement technique varying according to imaging modality, viewing conditions and the specific diagnostic task at hand [1,2].

Since digital radiographs are composed of a set of numbers arranged as a grid of rows and columns, the dentist can perform mathematical operations on these numbers to create a new image in which certain characteristics are enhanced, thus making interpretation of the image easier [3].

The most common image enhancement algorithms are based on linear adjustments such as brightness and contrast adjustment, and negative mode. Other algorithms are based on non-linear adjustments such as gamma correction [4-10].

Digital radiography has created a growing opportunity for computer-aided diagnostic tools that enable dentists to obtain more information from dental digital radiography than is possible with the unaided eye, leading to improved patient care [11].

Based on the use of image processing methods, various digital images with different image characteristics can be obtained for the same structure. Generic image enhancement methods can improve the diagnostic performance and subjective image quality in one task but does not always lead to a better outcome. For example, increasing the contrast between enamel and dentin might improve diagnosing

*Address correspondence to this author at the Department of Oral and Maxillofacial Radiology, Lebanese University, Beirut, Lebanon; Tel/Fax: +9611446413; E-mail: Ibrahim.nasseh@gmail.com interproximal caries but excess of contrast can cause false positive diagnosis of caries and will definitely create an unfavorable image for the diagnosis of periodontal diseases [12].

A new digital image processing algorithm is being developed. It is based on a 5th degree polynomial model, where grey values are converted to thickness values. By applying this algorithm, equal steps in object thickness will be perceived as equal steps in brightness change when digital radiographs are viewed.

Relating the changes of grey values to changes of absorber thicknesses gives this algorithm the advantage of using the examined structures as their own reference making this type of processing a more task/patient based enhancement method [13].

The purpose of this study is to compare the detected number of holes on a step wedge on images resulting from the application of the 5th degree polynomial model compared to the images resulting from the application of linear enhancement.

If proven effective, this enhancement method will be tested in pre-clinical/in vitro studies to assess its effectiveness in improving the detection of interproximal caries, root fracture and periodontal lesions by using a task specific calibration proper to each one of the latter situations.

\section{MATERIAL AND METHODS}

The test object was a customized 10-step aluminum step wedge at $1.5 \mathrm{~mm}$ increment and with five holes randomly drilled on each step at a depth varying from 0.2 to $1 \mathrm{~mm}$ (Fig. 1). The digital receptor used was a Size 2 Schick $33^{\circledR}$ (Sirona Dental Systems, Inc. Long Island City, NY, USA). 
The radiographs were exposed with the Planmeca Intra (Plane mcaOy, Helsinki, Finland)intraoral X-ray machine operating at 63,66 and $70 \mathrm{kVp}$ at $8 \mathrm{~mA}$, with 5 exposure times per $\mathrm{kVp}$ : from $0.016 \mathrm{~s}$ to $0.064 \mathrm{~s}$ totaling 15 exposures. The distance between source and receptor was fixed at 12 inches $(30 \mathrm{~cm})$.



Fig. (1). Step-wedge with holes used in the study.

The original 12-bit raw data was located in the image root folder. The images were transferred as tagged image file format files (TIFF) to a personal computer with a Microsoft Windows ${ }^{\circledR} 7$ operating system.

The software used for image analysis was Image ${ }^{\circledR}(\mathrm{Na}-$ tional Institute of Health, Bethesda, MD) and Microsoft Excel $^{\circledR}$ (Seattle, WA). Image ${ }^{\circledR}$ provided the numerical values from the original images of the step wedge and Microsof $\mathrm{t}^{\circledR}$ Excel $^{\circledR}$ provided the regression formulas and was used for converting the grey values to aluminum thickness values.

Images were opened in Image $^{\circledR}$, and from each image the histogram of a $100 \times 300$ pixel region of interest from each step was evaluated, and the mean grey value from this histogram was exported to Microsoft Excel $^{\circledR}$ and plotted against the thickness of its corresponding step. This generated the 5th degree polynomial fit (Poly) representing the change in grey values relative to the increasing step thickness. All images were converted based on this model.

The images were viewed as "original images" (Original), automatically adjusted brightness and contrast images (B/C), auto equalized images (Equalized) and images adjusted based on the 5th degree polynomial fit. A total of 60 images were viewed independently by six observers: Two Oral and Maxillofacial Radiologist (OMR) faculty, two OMR Residents and two Advanced Education in General Dentistry (AEGD) residents. The observers were asked to count the numbers of holes visible on each image. The reading was repeated after 2 weeks (Fig. 2).

\section{Statistical Analysis}

Data were analyzed using analysis of variance (ANOVA) for repeated measurements [14]. Observer classification was tested against a between-observer error; method, exposure time, and $\mathrm{kVp}$ were tested against a within-observer error. Comparisons among means following the ANOVA were Bonferroni adjusted. Residual analyses indicated that the data were in reasonable accord with the assumptions underlying the analysis of variance.
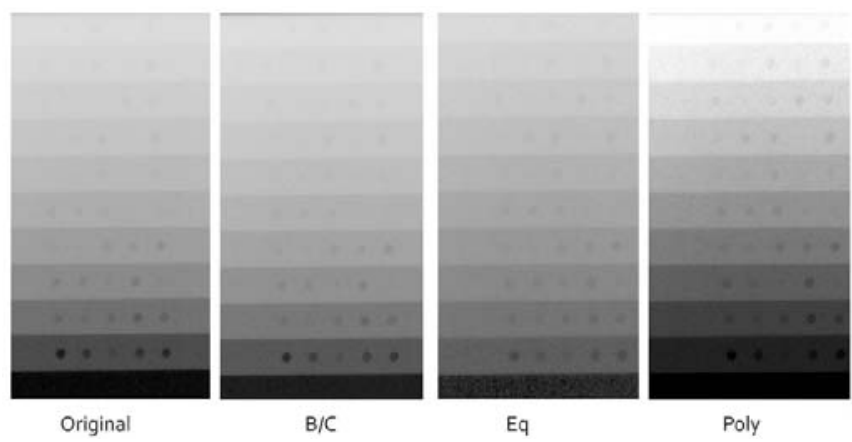

Fig. (2). Images compared on exposure settings: $66 \mathrm{kVp}$ and $0.04 \mathrm{~s}$

\section{RESULTS}

There was no significant difference $(\mathrm{P}=0.5017)$ between the observer classifications (OMR Faculty, OMR resident, and AEGD resident). Furthermore, there was no significant difference between the first and second reading by the observers $(\mathrm{P}=0.1217)$.

The average counts by Method, Exposure time and $k \mathrm{Vp}$, averaged over Observer and Reading, are shown in Fig. (3). There was a significant three-factor interaction among Method, Exposure time, and $\mathrm{kVp}$. This three factor interaction indicated that the difference between Methods varied with Exposure time and $\mathrm{kVp}$. Although differences among Methods vary, the overall pattern was the same, that is, "Poly" results in the highest counts, "Original" in the lowest counts, with "B/C" and "Equalized" intermediate.

Fig. (4) shows the average counts by Method and Exposure time, averaged over kVp, Observer, and Reading. The two-factor interaction was statistically significant. This is shown in the figure in which the differences between Methods vary with Exposure time. As indicated in the figure, average counts using Poly were significantly higher than obtained with the other methods for all exposure times. Average counts were lower using Original compared to the B/C and Equalized at all exposure times other than 0.064. B/C and Equalized were not significantly different at exposure times 0.016 and 0.04 ; at exposure time $0.025,0.05$, and 0.064 , Equalized was significantly higher than $\mathrm{B} / \mathrm{C}$.

Fig. (5) shows the average counts by Method and $k V p$, averaged over Exposure time, Observer, and Reading. The two-factor interaction was statistically significant. However, the changes with $\mathrm{kVp}$ indicate that the effect of $\mathrm{kVp}$ was small relative to the size of the effect of Method.

Fig. (6) shows the average counts by Exposure time and $\mathrm{kVp}$, averaged over Method, Observer, and Reading. The two-factor interaction was statistically significant. However, the changes with $\mathrm{kVp}$ again indicate that the effect of $\mathrm{kVp}$ was small relative to the size of the effect of Exposure time.

Within each set of observations of Exposure time, $k \mathrm{Vp}$, Observer, and Reading, we ranked the number of counts 


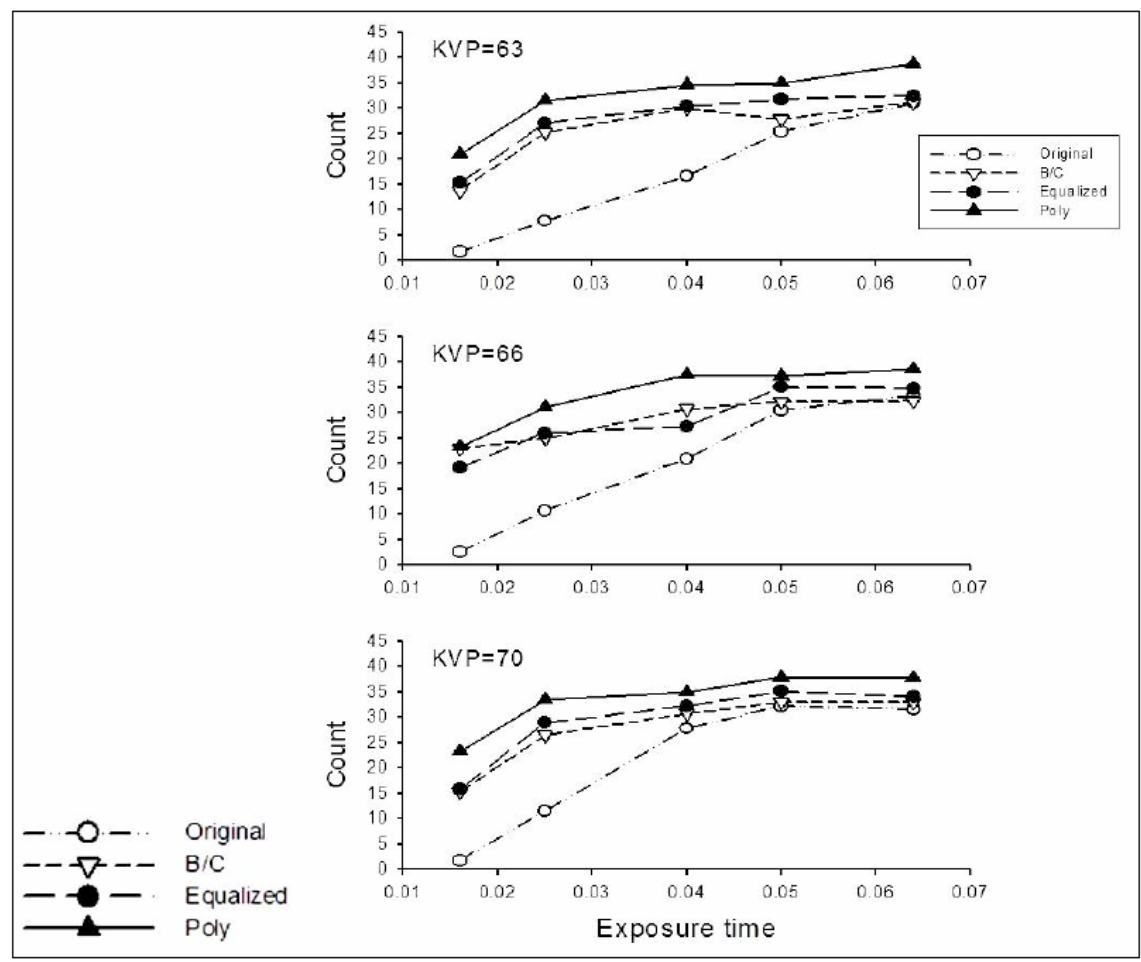

Fig. (3). Mean number of counts by Method, Exposure time and kVp averaged over Observer and Reading.

obtained by each of the four methods from 1 (lowest) to 4 (highest). Observations of the same count were assigned the average rank. Clearly use of Poly achieved the highest number of counts and Original the lowest; B/C and Equalized were intermediate. These results are in line with the results obtained by examining the average counts.

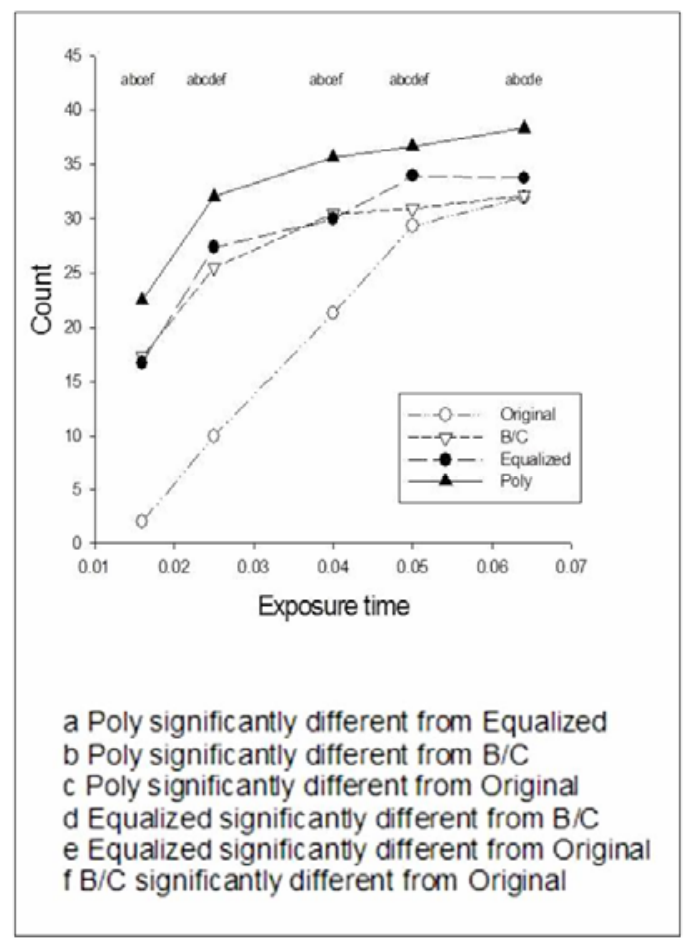

Fig. (4). Mean number of counts by Method and Exposure, averaged over kVp, Observer and Reading.

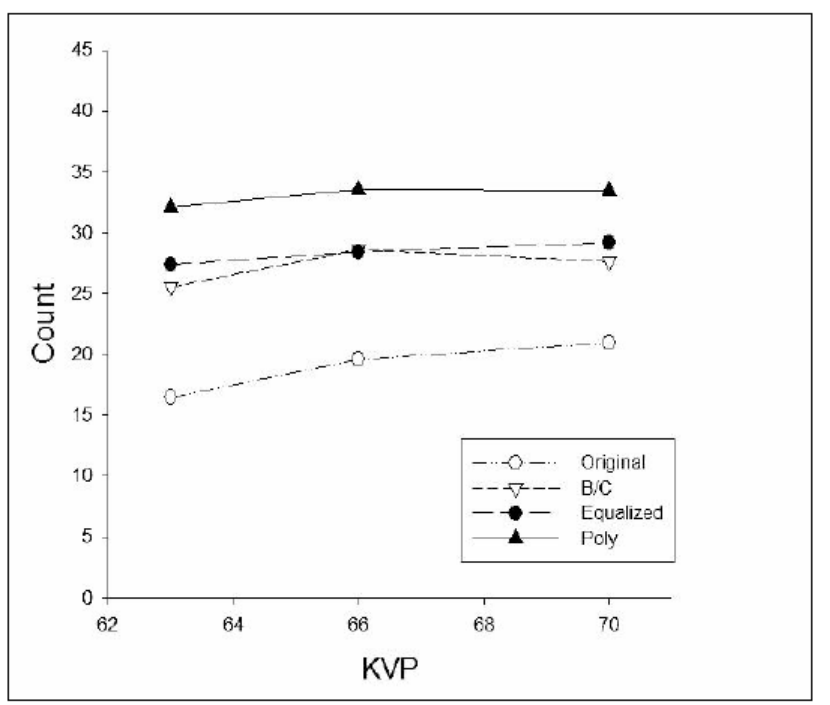

Fig. (5). Mean number of counts by Method and $\mathrm{kVp}$, averaged over Exposure time, Observer and Reading.

\section{DISCUSSION}

In the present study, images resulting from enhancement based on a $5^{\text {th }}$ degree polynomial model was compared to non-enhanced images and images enhanced with adjustment of brightness and contrast and with auto equalization. The results show that images enhanced with the polynomial model enabled higher number of visible holes on the step wedge. It was noted that the application of contrast/brightness and histogram equalization performed better than the original unenhanced images. This follows the results from Li et al., and Alpoz et al. [15, 16]. 


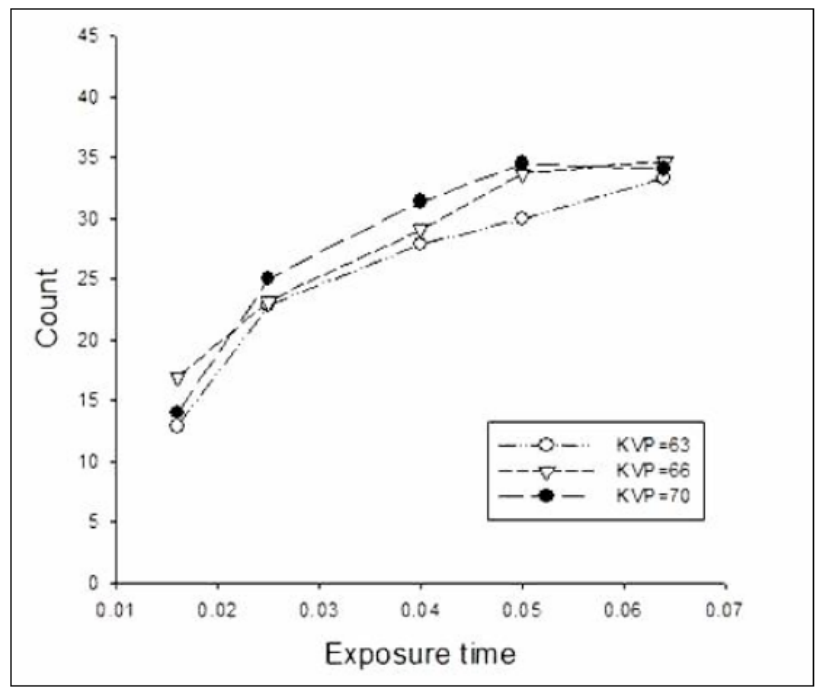

Fig. (6). Mean number of counts by Exposure time and kVp, averaged over Method, Observer and Reading.

As can be expected, higher number of holes was observed on images obtained with higher $\mathrm{kVp}$ and longer exposure times before enhancement. When interactions are present, we should be extremely careful in presenting effects averaging over the factors involved in the interactions. Because of the statistically significant three-factor interactions above, this rule applies and we must be cautious in presenting the data as we have done in Figs. (4-6). However, it was evident that no further benefit could be achieved with the use of processing algorithms, mainly the "poly" algorithm, when the $\mathrm{kVp}$ was increased. This result means that none of the processing algorithms used in this study had a negative impact on the counting visible holes, since no degradation in image content was noticed with any of the processing algorithms and that both the effects of Method and Exposure time are much larger than the effect of $\mathrm{kVp}$.

It has been shown by many studies that contrast/brightness enhancement is effective for the perception of small contrast details. Similar findings were observed in this study [17-19]. The histogram equalization algorithm converts the histogram of an original image to an equalized histogram; in other words, it changes the use of available grey values and the characteristics of the histogram [3]. The image parts with more frequency variation will be more enhanced, while parts of the image with less frequency will be neglected.

Our results clearly demonstrated that histogram equalization is effective in increasing the number if counted holes even at lower exposures. This also was proven in previous studies [16, 20-23].

The "Poly" algorithm showed the maximum number of counted holes, both the lowest and widest exposure ranges. By re-distributing the grey levels based on the actual thickness of each step with holes, a uniform re-distribution of grey levels in the output range is obtained, and the contents of the image accordingly became clearer to the observers [13].
The difference between number of holes detected at $0.05 \mathrm{~s}$ and $0.064 \mathrm{~s}$ is not statistically significant in the images processed with the polynomial algorithm; this means that almost a $20 \%$ decrease in exposure time did not affect the image quality, this is consistent with other studies done in the medical field showing that low dose images can be enhanced with post processing, making exposure at high doses unnecessary [23].

\section{CONCLUSION}

This study compared the number of visible holes on a step wedge with three different image processing algorithms. The goal was finding the best enhancement algorithm to reliably increase the number of visible holes. Among the algorithms used, the $5^{\text {th }}$ degreepolynomial model provided subjectively superior images. Further clinical studies needs to be done to confirm the correlation between perceptibility of subtle changes in anatomic structures (e.g. caries, root canal and periapical lesion) and diagnostic accuracy. This might result in reducing the patient's dose and in simplifying observer performance. This might also facilitate in vivo evaluations of new applications in digital imaging.

\section{CONFLICT OF INTEREST}

The authors confirm that this article content has no conflict of interest.

\section{ACKNOWLEDGEMENTS}

Declared none.

\section{REFERENCES}

[1] Analoui M. Radiographic image enhancement. Part I: spatial domaintechniques. Dentomaxillofac Radiol 2001; 30(1): 1-9.

[2] Mol A. Image processing tools for dental applications. Dent Clin North Am 2000; 44(2): 299-318.

[3] Van der Stelt PF. Better imaging: the advantages of digital radiography. J Am Dent Assoc 2008; Suppl 139: 7S-13S

[4] Analoui M. Radiographic digital image enhancement. Part II: transform domain techniques. Dentomaxillofac Radiol 2001; 30(2): 65 77.

[5] Braunstein EM, Capek P, Buckwalter K, Bland P, Meyer CR Adaptive histogram equalization in digital radiography of destructive skeletal lesions. Radiology 1988; 166(3): 883-5.

[6] Hayakawa Y, Kitagawa H, Wakoh M, Kuroyanagi K, Welander U. Assessing the image quality of a CCD-based digital intraoral radiography system: application of perceptibility curve test. Bull Tokyo Dent Coll 2000; 41(1): 9-14.

[7] Kruger RP, Hall EL, Dwyer SJ, Lodwick GS. Digital techniques for image enhancement of radiographs. Int J Biomed Comput 1971; 2(3): 215-38.

[8] Stahl M, Aach T, Dippel S. Digital radiography enhancement by non linear multi scale processing. Med Phys 2000; 27(1): 56-65.

[9] Welander U, Nelvig P, Tronje G, et al. Basic technical properties of a system for direct acquisitionof digital intraoral radiographs. Oral Surg Oral Med Oral Pathol 1993; 75(4): 506-16.

[10] Yoshiura K, Stamatakis HC, Welander U, et al. Physical evaluation of a system for direct digital intra-oralradiography based on a charge-coupled device. Dentomaxillofac Radiol 1999; 28(5): 27783.

[11] Tracy KD, Dykstra BA, Gakenheimer DC, et al. Utility and effectiveness of computer-aided diagnosis of dental caries. Gen Dent 2011; 59(2): 136-44

[12] Jin-Woo C, Won-Jeong H, Eun-Kyung K. Image enhancement of digital periapical radiographs according to diagnostic tasks. Imaging Sci Dent 2014; 44(1): 31-5. 
[13] Geha H, Bechara B, Faddoul T, Noujeim M. A mathematical model relating changes of grey values to changes of thicknesses of a step wedge. Dentomaxillofac Radiol 2013; 42(1): 50719185.

[14] Winer BJ. Statistical principles in experimental design. $2^{\text {nd }}$ ed. McGraw-Hill: New York 1971; p. 924.

[15] Li G, Welander U, Yoshiura K, Shi XQ, McDavid WD. Perceptibility curve test for digital radiographs before and after correction for attenuation and correction for attenuation and visual response. Dentomaxillofac Radiol 2003; 32(6): 372-8.

[16] Alpoz E, Sogur E, BaksiAkdeniz BG. Perceptibility curve test for digital radiographs before and after application of various image processing algorithms. Dentomaxillofac Radiol 2007; 36(8): 490-4.

[17] Walker A, Horner K, Czajka J, Shearer AC, Wilson NH. Quantitative assessment of a new dental imaging system. Br J Radiol 1991; 64(762): 529-36.

[18] Møystad A, Svanaes DB, van der Stelt PF, et al. Comparison of standard and task-specific enhancement of Digora storage phosphor images for approximal caries diagnosis. Dentomaxillofac Radiol 2003; 32(6): 390-6.
[19] Møystad A, Svanaes DB, Risnes S, Larheim TA, Gröndahl HG. Detection of approximal caries with a storage phosphor system. A comparison of enhanced digital images with dental X-ray film. Dentomaxillofac Radiol 1996; 25(4): 202-6.

[20] Heo MS, Choi DH, Benavides E, et al. Effect of bit depth and kVp of digital radiography for detection of subtle differences. Oral Surg Oral Med Oral Pathol Oral Radiol Endod 2009; 108(2): 278-83.

[21] Shi XQ, Li G, Yoshiura K, Welander U. Perceptibility curve test for conventional and colour-coded radiographs. Dentomaxillofac Radiol 2004; 33(5): 318-22.

[22] Kositbowornchai S, Basiw M, Promwang Y, Moragorn H, Sooksuntisakoonchai N. Accuracy of diagnosing occlusal caries using enhanced digital images. Dentomaxillofac Radiol 2004; 33(4): 23640.

[23] Egbe NO, Heaton, B, Sharp PF. A simple phantom study of the effects of dose reduction (by $\mathrm{kVp}$ increment) below current dose levels on CR chest image quality. Radiography 2010; 16: 327-32.

(C) Geha et al.; Licensee Bentham Open.

This is an open access article licensed under the terms of the Creative Commons Attribution Non-Commercial License (http://creativecommons.org/licenses/by-nc/3.0/) which permits unrestricted, non-commercial use, distribution and reproduction in any medium, provided the work is properly cited. 